\title{
SÍNDROME DE QUEMARSE POR EL TRABAJO Y SATISFACCIÓN LABORAL COMO PREDICTORES DE CALIDAD DE LA ATENCIÓN DE ENFERMERÍA HOSPITALARIA ${ }^{1}$
}

\section{BURNOUT SYNDROME AND LABOR SATISFACTION AS PREDICTORS OF QUALITY OF THE HOSPITAL NURSING ATTENTION}

\author{
Paz Eugenia Soto Fuentes* \\ Silvia Barrios Araya ${ }^{* *}$ \\ Yerko Molina Muñoz ${ }^{* * *}$
}

\begin{abstract}
RESUMEN
Objetivo: Establecer si existe relación entre el Síndrome de Quemarse en el Trabajo (SQT) y satisfacción laboral con la calidad de la atención brindada por equipos de enfermeras en un hospital público de alta complejidad de la Región Metropolitana, Chile. Material y método: Estudio observacional, descriptivo, correlacional de tipo ecológico-mixto. La muestra fueron 35 enfermeras, distribuidas en 12 turnos, de servicios de Medicina y Cirugía y 402 pacientes en etapa de egreso o recuperación de su cuadro agudo hospitalizados en estos servicios. Instrumentos: Cuestionario para Evaluación del Síndrome de Quemarse en el Trabajo, Encuesta de Satisfacción Laboral Hospitalaria y Escala SERVQUAL. Resultados: Las enfermeras presentaron niveles medios de satisfacción laboral (Media=3,18 DS=0,38); el resultado global de SQT mostró valores bajos (Media=23,62 DS=9,63) y en la calidad de atención percibida por los usuarios se observó que todas las expectativas fueron superiores a 6,5. La mayoría de las dimensiones de satisfacción laboral evidenciaron correlaciones estadísticamente significativas negativas con las dimensiones de SQT. La relación entre satisfacción laboral global de los equipos de enfermeras(os) y calidad de atención global percibida por los usuarios no mostró una correlación significativa, presentándose relaciones estadísticamente positivas entre algunas de sus dimensiones Conclusión: La relación entre SQT y satisfacción laboral con la calidad de la atención muestra hallazgos que no son concluyentes. Las dimensiones de satisfacción laboral global presentaron una correlación positiva baja con calidad de atención y entre SQT global y calidad de atención percibida se presentó una relación muy baja y negativa con calidad de atención global.
\end{abstract}

Palabras clave: Agotamiento profesional, satisfacción laboral, personal de enfermería en hospital, atención hospitalaria, calidad de la atención de salud.

\begin{abstract}
Objective: To establish whether there is a relationship between the Burnout Syndrome and job satisfaction with the quality of care provided by teams of nurses in a highly complex public hospital in the Metropolitan Region of Chile. Method: Descriptive and correlational observational study of mixed-ecological type. The studied sample Chile.

${ }^{1}$ Fuente de Financiamiento Proyecto Puente 24/2014 Vicerrectoría de Investigación Pontificia Universidad Católica de

${ }^{*}$ Enfermera Matrona, Profesor Titular, Escuela de Enfermería Pontificia Universidad Católica de Chile, Santiago-Chile. Email: pesoto@uc.cl

${ }^{* *}$ Enfermera, Profesor Asociado. Escuela de Enfermería Pontificia Universidad Católica de Chile. Email: sbarrios@uc.cl

${ }^{* * *}$ Estadístico, Profesor Asistente. Escuela de Psicología Pontificia Universidad Católica de Chile. Email: ypmolina@uc.cl
\end{abstract}


was 35 nurses, distributed in 12 shifts, of the services of Medicine and Surgery and 402 patients in a stage of discharge or recovery of their acute disease hospitalized in these services. Measures: For the measurement of the burnout syndrome, Spanish Burnout Inventory was used; to measure job satisfaction the hospital satisfaction survey was applied and the scale SERVQUAL was used to measure of the user satisfaction. Results: Nurses presented average levels of job satisfaction (Mean=3.18 DS=0.38; regarding the SQT, the overall result shows low values (Mean=23.62 DS=9.63) and with respect to the quality of attention perceived by users, it can be seen that all expectations tend to be higher than 6.5. Most of the factors of job satisfaction showed statistically significant negative correlations with the SQT factors. The relationship between the overall job satisfaction of the nurses' teams and the quality of global attention perceived by the users does not present a significant correlation, yet statistically positive relationships between some of their factors were found. Conclusion: The relationship between SQT and job satisfaction with the Quality of care presents findings that are inconclusive, since the correlations present different levels of statistical significance among the factors. Global job satisfaction factors showed a low positive correlation with quality of care and the relationship between global burnout syndrome and perceived quality of care presented very low and negative relationship with global quality of care.

Key words: Professional burnout, job satisfaction, hospital nursing staffs, hospital care, quality of health care

Fecha recepción: 28/01/16 Fecha aceptación: 03/10/17

\section{INTRODUCCIÓN}

El mundo laboral ha experimentado cambios en el contexto sociocultural, pues las exigencias y los requisitos del puesto de trabajo han causado problemas de salud en las personas, entre los que destaca el Síndrome Quemarse por el Trabajo (SQT), propio de los profesionales que otorgan servicios (1). Las organizaciones se preocupan del bienestar y de la salud laboral porque se reconoce que su ausencia repercute en la eficacia, la calidad de vida en el trabajo y el ausentismo laboral, cuyas consecuencias implican mayores costos económicos y sociales (2).

Como antecedente, es relevante mencionar que el Síndrome de Burnout, traducido al español por Gil-Monte como Síndrome de Quemarse por el Trabajo (SQT) (1), es un fenómeno que comenzó a ser investigado en los Estados Unidos a principios de los años 70, para explicar el proceso de deterioro en los cuidados y en la atención profesional a los usuarios de las organizaciones de salud. Conceptualmente, el referente más importante en el tema es Maslach con su modelo multi- funcional (3), quien, en 1981, lo define como un síndrome psicológico de agotamiento emocional, despersonalización y reducción de la realización personal. Posteriormente, Maslach plantea que el foco del fenómeno está en el proceso de erosión psicológica y social de una exposición crónica al estrés, es decir, el problema no es de las personas, sino de la estructura y funcionamiento del ambiente social en el cual trabajan (4). A su vez, Gil-Monte define SQT como una respuesta psicológica al estrés laboral crónico, de carácter interpersonal y emocional, caracterizado por un deterioro cognitivo y afectivo. El deterioro cognitivo se manifiesta por pérdida de la ilusión por el trabajo y desencanto profesional. El deterioro afectivo se manifiesta por agotamiento emocional y físico y por actitudes y/o conductas negativas hacia los clientes y hacia la organización. Todos estos sentimientos a veces están acompañados de culpa (1).

Aunque las investigaciones muestran una relación positiva entre SQT y satisfacción laboral, la comprensión de cómo limitar el impacto de las dimensiones del estrés sigue siendo ambigua y se desconoce la relación y 
efecto entre SQT y los resultados en la calidad de atención otorgada (2, 5-8). No obstante, se ha encontrado que a mayor insatisfacción en el trabajo por parte del equipo de enfermeras, menor es la calidad de la atención $(9,10)$.

En este contexto, es importante estudiar el SQT y la satisfacción laboral porque, aunque se consideran dos constructos multidimensionales que representan respuestas afectivas diferentes al trabajo, están altamente relacionadas entre sí. Una característica importante del SQT que se relaciona con la satisfacción laboral es la despersonalización, es decir, sentimientos y actitudes de carácter negativo en la habilidad de realizar el trabajo y de relacionarse con las personas que se atienden (2); es un aspecto que, respecto de la satisfacción, describe el grado de afecto positivo hacia el trabajo y sus componentes (6).

Estudios realizados en Australia, Canadá, Estados Unidos, Jordania e Irán describen otras dimensiones que pueden influir en la satisfacción laboral, entre los que se encuentran: la inflexibilidad en el horario de trabajo, las políticas organizativas y el reconocimiento profesional (4), aspectos que, en los equipos de enfermería, se consideran clave ante la decisión de abandonar el trabajo como consecuencia de una insatisfacción laboral (5).

Las condiciones laborales y las características del tipo de trabajo desarrollado convierten a las enfermeras de los servicios clínicos en un grupo de riesgo de presentar SQT y figuran como causales la escasez de personal, la carga de trabajo, la presión del tiempo, la falta de previsibilidad, el acoso laboral y los estilos de dirección $(7,8)$. Asimismo, el propio paciente también se convierte en factor de SQT en situaciones tales como: la agresividad, el enfrentamiento a la muerte, el dolor, el sufrimiento o el hacer frente a los familiares de pacientes desafiantes (7-9). Todas situaciones que producen efectos perjudiciales en la salud física y psicológica de enfermeras(os), vinculadas a errores clínicos, bajas tasas de retención del personal, altas tasas de ausentismo y agotamiento precoz (10-16).
Por su parte, la satisfacción del usuario es entendida como el grado de cumplimiento, por parte del sistema de salud, de las expectativas del usuario en relación a los servicios que este ofrece y es un indicador de la excelencia en la calidad de atención (17). Aunque en Chile el nivel de satisfacción de los usuarios respecto de la atención recibida es relativamente buena y la atención hospitalaria es la que refleja el mayor nivel de satisfacción (18), también es importante considerar que en el personal de enfermería se han encontrado niveles altos del SQT (35\%) (16), prevalencia importante de considerar si se sabe que la satisfacción de los usuarios del sistema de salud, como medida de calidad de la atención, está relacionada con equipos de atención que reportan satisfacción laboral y en donde no hay presencia de SQT.

Según lo descrito, la presente investigación tuvo como propósito establecer si existe relación entre SQT y satisfacción laboral de las enfermeras con la calidad de atención de los usuarios, medida a través de la satisfacción del usuario en un hospital público de alta complejidad de la Región Metropolitana (RM).

\section{MATERIAL Y MÉTODO}

Tipo de estudio: Estudio observacional correlacional descriptivo de tipo ecológico-mixto, realizado en un hospital público de alta complejidad de la Región Metropolitana de Chile, entre los meses de julio a septiembre del año 2015. Se aplicó diseño ecológico-mixto por la necesidad de medir las variables independientes en el equipo de enfermeras y la dependiente en cada paciente de manera individual. Lo anterior se debe a que no es posible establecer una relación, uno a uno, entre paciente y enfermera porque el paciente es atendido por un equipo y por tanto su evaluación no es sobre una enfermera individual. 
Unidades de análisis: Equipo o grupo de turno de enfermeras/os y paciente hospitalizado, atendido por uno de los grupos de enfermeras, en los servicios de medicina y cirugía. Se excluyeron pacientes en etapa aguda de su enfermedad y equipos de enfermeras/os de servicios de alta complejidad como cuidados intermedios, críticos, atención transitoria y urgencia.

Muestra: Para el cálculo del tamaño de la muestra no probabilística y por conglomerados, se consideró un error de tipo I a priori de $0,05(\alpha=0,05)$, un poder estadístico teórico de 0,8 con un tamaño de efecto pequeño. Participaron del estudio los 12 equipos de enfermeras/os de los servicios de medicina y cirugía. El nivel de participación fue de $74,4 \%$, contando con 35 profesionales que cumplieron con los requisitos de inclusión y aceptaron participar. Del total de pacientes hospitalizados en ese período (julio a septiembre 2015), atendidos por el equipo de enfermeras/os, participaron 402 pacientes que estaban en etapa de egreso o recuperación de su cuadro agudo, alcanzando un $10,2 \%$ muestral.

Recolección de datos: Para la recolección de datos se utilizaron los siguientes instrumentos:

1) Encuesta de variables sociodemográficas y laboral: cuestionario de elaboración propia, referida a datos de tipo personal (sexo, edad, estado civil, número y edad de los hijos), de filiación y laboral de las enfermeras (años de ejercicio profesional, años de trabajo en la institución, servicio en que trabaja, horario de trabajo, cargo que ocupa, tipo de contrato, régimen de salud y satisfacción con su remuneración).

2) Encuesta de Satisfacción Laboral hospitalaria de Font Roja, instrumento de medida de la satisfacción laboral en el medio hospitalario de Aranaz y Mira (19), quienes autorizaron su uso. Consta de 23 ítems agrupados en 7 dimensiones: "Satisfacción en el trabajo", "Presión en el trabajo", "Relación personal", "Distensión en el trabajo" "Adecuación para el trabajo", "Control sobre el trabajo" y "Variedad de la tarea". Fue validado en el contexto mexicano en donde obtuvo un Alfa de Cronbach $=0,80$ (20). Las respuestas para cada pregunta están en formato Likert y contemplan puntajes de 1 a 5 , siendo 1 menor satisfacción y 5 el puntaje de mayor satisfacción. En este estudio una puntuación menor a 2,5 se consideró baja, un puntaje entre 2,5 y 3,5 puntos se consideró medio y alto, valores mayores a 3,5. Para los puntajes por factor se promedió las respuestas de los sujetos en las preguntas correspondientes a cada factor, lo que dio un puntaje de 1 a 5 para cada factor, donde a mayor puntaje mayor satisfacción con el trabajo, considerando como valor medio el puntaje de 3 . Además de los puntajes por factor se realizó un puntaje global de satisfacción laboral que se calculó utilizando el promedio simple de las respuestas a la totalidad de las preguntas del instrumento.

3) Cuestionario para evaluación del Síndrome de Quemarse en el Trabajo (CESQT) de Gil- Monte, instrumento de uso libre validado en Chile con Alfa de Cronbach superior a 0,70 (21), consta de 20 preguntas en escala Likert puntuadas de 0 a 4 y distribuidas en 4 dimensiones: "Ilusión por el trabajo", "Desgaste psíquico" "Indolencia" y "Culpa". Los puntajes globales en esta escala tienen como límite el 0 para los puntajes bajos y el 80 para los altos. Para calcular los puntajes globales se categorizaron los ítems correspondientes al factor de "ilusión por el trabajo", de tal manera que la escala siempre se pudiera interpretar en una correlación de dirección positiva, es decir, a mayor puntaje, mayor SQT. 
Para interpretar estos resultados se definió como bajo nivel a los puntajes promedios inferiores al punto medio de cada escala global y por dimensión. Para hacer comparables las distintas dimensiones del SQT, se tomaron los puntajes brutos para cada escala y se dividieron por el $\mathrm{N}^{\circ}$ de ítems. Esto arrojó un promedio que va de 0 a 4 para todas las escalas, siendo 2 el valor medio. Además, para comparar las dispersiones se compararon los coeficientes de variación.

4) Para valorar la Satisfacción usuaria se utilizó la Escala multidimensional SERVQUAL (22), que evalúa la calidad del servicio a partir de la diferencia entre las expectativas y percepciones del usuario, por lo que se considera como la más apropiada para evaluar la calidad del servicio de enfermería. Constituida por dos subescalas, una de expectativas y otra de percepciones, posee cinco dimensiones: a) elementos tangibles: apariencia del personal, facilidades físicas, equipo e información; b) confiabilidad: habilidad para proporcionar el servicio prometido con precisión y eficacia; c) responsabilidad: habilidad para ayudar y proporcionar el servicio rápidamente; d) seguridad: habilidad para inspirar confianza y credibilidad; y e) empatía: atención individual y personalizada. Utiliza dos cuestionarios de 22 ítems cada uno y cada ítem es evaluado por medio de una escala de diferencial semántico que varía de 1 a 7 , siendo 1 totalmente de acuerdo y siete totalmente en desacuerdo, con el 4 como valor neutro. Como resultante, la calidad percibida del servicio es igual a la resta entre los puntajes de expectativas menos los puntajes de las percepciones. Así, se generan puntajes entre - (menos) 7 y 7 y la interpretación es que a menor brecha (diferencia) mayor satisfacción. Esta manera de puntuar facilita el análisis de los resultados ya que su expre- sión global tiende a ser en números positivos. La escala, de uso libre validada en Chile, ha presentado un Alfa de Cronbach $=0,895$ tanto para expectativas como para percepciones (23) y en Colombia la validez fue de 0,9075 para las expectativas y 0,9377 para las percepciones (24).

Procedimiento: Tanto los pacientes como las enfermeras/os se reclutaron en forma individual. Se realizó una reunión previa con enfermeras/os coordinadores o supervisores de los servicios para informarles del estudio. A los pacientes se les solicitó el consentimiento informado cuando su condición de salud (estar en etapa de egreso o recuperación de su cuadro agudo) les permitió participar.

Consideraciones éticas: El estudio fue aprobado por el Comité Ético-Científico de la Facultad de Medicina de la Pontificia Universidad Católica de Chile ( $\left.\mathrm{N}^{\circ} 14-138\right)$ y el Comité Ético-Científico del Servicio de Salud ( $\mathrm{N}^{\circ}$ 019892). Los ayudantes del proyecto fueron entrenados para reclutar, recibir las encuestas y mantener la confidencialidad de los participantes. A aquellos pacientes que aceptaron participar se les entregó el cuestionario autoaplicado para evaluar Satisfacción usuaria SERVQUAL. Una vez que el instrumento era completado se depositaba en una urna sellada.

Análisis estadístico: Para describir la muestra de enfermeras(os) y/o los puntajes en las distintas escalas se utilizaron medidas de tendencia central, dispersión, percentiles y coeficiente de asimetría. Se calcularon los coeficientes de variación para tener medidas de comparación de la dispersión entre distintas dimensiones. Para la correlación entre SQT y satisfacción laboral con la calidad de atención se utilizó la medida no paramétrica de Spearman debido a que la prueba de Kolmogorov Smirnov reveló que las variables no presentaron una distribución normal. 


\section{RESULTADOS}

Perfil sociodemográfico y laboral de enfermeras(os): Los equipos de enfermeras(os) tenían 33 años de edad promedio (DS 8,2), la mayoría mujeres $(97,1 \%)$, solteras $(54,3 \%)$ y sin hijos $(51,4 \%)$. El perfil laboral evidenció que la mayoría $(42,9 \%)$ tenía entre 1 y 3 años de ejercicio laboral. Respecto al tipo de contrato, la mayoría $(45,7 \%)$ tenía contrato a plazo fijo y junto a los que tenían contrato a honorario muestran una mayor proporción de satisfacción con el sueldo (60\%), comparado con el descontento que expresa el 17,1\% que está contratado indefinidamente.

Satisfacción Laboral: Las enfermeras presentan en general niveles medios de satisfacción laboral, con promedios para las 7 dimensiones cercanos a la mitad del puntaje total posible (entre 2,3 y 3,5 de un total de 5 puntos). Las dimensiones que presentan los puntajes más altos son "adecuación para el trabajo", "relación personal" y "satisfacción en el trabajo". Las dimensiones que presentan los puntajes más bajos fueron "distensión en el trabajo" "presión en el trabajo" y "control sobre el trabajo" (Tabla 1).

Síndrome de Quemarse por el Trabajo: El resultado global muestra valores bajos, con puntajes mínimos de 1 y máximo de 50 puntos (Tabla 2). El "desgaste psíquico", que resulta de la respuesta a "me siento desgastado/a emocionalmente", es la dimensión en el que las enfermeras presentan el mayor nivel de SQT. Las dimensiones que presentan menor SQT son "ilusión por el trabajo", "indolencia" y "culpa".

Calidad de Atención percibida por los usuarios: $\mathrm{Al}$ analizar las expectativas y las percepciones de la calidad de atención percibida, se puede observar que las expectativas tienden a ser bastante altas, todas superiores a 6,5 de un máximo de 7. Al analizar las diferencias entre expectativas y percepciones, se puede observar que la brecha más pequeña se encuentra en la dimensión "seguridad", seguida de la dimensión "capacidad de respuesta”. Las dimensiones que presentan una mayor brecha $y$, por lo tanto, menor calidad de atención percibida corresponden a "confiabilidad" y "empatía" (Tabla 3).

Análisis correlacional: Satisfacción laboral de los equipos de enfermeras(os) y calidad de atención percibida: Se observa que el puntaje global y las dimensiones "satisfacción" y "presión" presentan una correlación positiva baja con la magnitud de la brecha para "elementos tangibles" (Rho $=0,122$ y 0,111 con $\mathrm{p}<0,05$ y 0,138 con $\mathrm{p}<0,001$, respectivamente), asimismo "control" presenta una correlación positiva y baja con las brechas de "calidad de atención globales" ( $R h o=0,106$; $\mathrm{p}<0,05)$ y con la dimensión "empatía" (Rho = $0,155 ; p<0,01)$. Las dimensiones de satisfacción laboral que más relaciones significativas presentan con calidad de atención son "variedad" y "distensión". El primero se relaciona de manera significativa, débil y negativa con cuatro de cinco dimensiones: "elementos tangibles" (Rho $=-0,115 ; \mathrm{p}<0,05)$, "confiabilidad" (Rho $=-0,106 ; \mathrm{p}<0,05)$, "capacidad de respuesta" (Rho $=-0,117 ; \mathrm{p}<0,05)$ y "seguridad" (Rho $=-0,112 ; p<0,05)$. El segundo, "distensión", se correlaciona de manera significativa, débil y positiva con todos las dimensiones de la calidad de atención (Tabla 4).

SQT y calidad de atención percibida: En la Tabla 5 se observa que el SQT global tiene una relación muy baja y negativa con calidad de atención global $(\mathrm{Rho}=-0,115 ; \mathrm{p}<0,05)$, con las dimensiones "empatía" (Rho = $-0,109 ; \mathrm{p}<0,05) \mathrm{y}$ "elementos tangibles" (Rho $=-0,141 ; p<0,01)$. Lo mismo ocurre con la dimensión de SQT "indolencia" con calidad de atención global (Rho $=-0,111 ; p<0,05)$, "empatía" (Rho $=-0,101 ; \mathrm{p}<0,05)$, "confiabilidad" (Rho $=-0,101 ; \mathrm{p}<0,05)$ y "seguridad" (Rho $=-0,105 ; p<0,05)$. Finalmente, 
la dimensión de SQT “culpa” presenta una asociación negativa y muy baja en casi todas las dimensiones de calidad de atención percibida por los usuarios, a excepción de la "capacidad de respuesta" dimensión con el que no presenta relación estadísticamente signifi- cativa. Se observa que "desgaste" no presenta ninguna asociación estadísticamente significativa con calidad de atención, es decir, para esta muestra el leve desgaste psíquico es independiente de la calidad de atención.

Tabla 1. Distribución de puntajes en Satisfacción laboral de enfermeras(os), Servicios de Medicina y Cirugía en Hospital de la RM, Chile.

\begin{tabular}{|c|c|c|c|c|}
\hline Dimensiones & Mínimo & Máximo & Media & DS \\
\hline 1. Satisfacción en el trabajo & 2 & 4,33 & 3,44 & 0,52 \\
\hline Presión en el trabajo & 1,25 & 4,25 & 2,69 & 0,65 \\
\hline 3. Relación personal & 2,67 & 4,67 & 3,54 & 0,58 \\
\hline 4. Distensión en el trabajo & 1 & 4,5 & 2,26 & 0,86 \\
\hline 5. Adecuación para el trabajo & 2,75 & 4,5 & 3,57 & 0,45 \\
\hline 6. Control sobre el trabajo & 1,67 & 5 & 3 & 0,72 \\
\hline 7. Variedad de la tarea & 2 & 5 & 3,31 & 0,83 \\
\hline Satisfacción Laboral Global & 2,26 & 4,17 & 3,18 & 0,38 \\
\hline
\end{tabular}

Tabla 2. Distribución de puntajes Cuestionario para Evaluación del Síndrome de Quemarse en el Trabajo (CESQT) en enfermeras(os), Servicios de Medicina y Cirugía, Hospital de RM de Chile.

\begin{tabular}{lcccrc}
\hline $\begin{array}{l}\text { Dimensiones de Síndrome de } \\
\text { Quemarse en el Trabajo }\end{array}$ & Mínimo & Máximo & Máximo posible* & Media & DS \\
\hline 1. Ilusión por el trabajo & 6 & 20 & 20 & 15,02 & 3,91 \\
2. Desgaste Psíquico & 1 & 14 & 16 & 7,52 & 3,44 \\
3. Indolencia & 0 & 17 & 24 & 5,64 & 3,73 \\
4. Culpa & 0 & 13 & 20 & 5,47 & 3,23 \\
\hline SQT Global & 6 & 50 & 80 & 23,62 & 9,63 \\
\hline
\end{tabular}

*Único factor que se interpreta negativamente, es decir mayores puntajes representan menos SQT.

**El máximo posible es el máximo valor para la dimensión de burnout que la escala permite.

Tabla 3. Distribución de puntajes de satisfacción usuaria para medir Calidad de atención en pacientes servicios de Medicina y Cirugía, Hospital de RM de Chile.

\begin{tabular}{lccc}
\hline Dimensiones & Expectativa & Percepción & Diferencia \\
\hline Empatía & 6,595 & 5,7886 & 0,8064 \\
Elementos Tangibles & 6,5454 & 5,7677 & 0,7776 \\
Confiabilidad & 6,5919 & 5,7724 & 0,8195 \\
Capacidad de Respuesta & 6,5371 & 5,8106 & 0,7265 \\
Seguridad & 6,7129 & 6,1784 & 0,5344 \\
\hline Total & 6,5988 & 5,8547 & 0,744 \\
\hline
\end{tabular}


Tabla 4. Satisfacción laboral y Calidad de atención de equipos de enfermería, Servicios de Medicina y Cirugía, Hospital de RM de Chile.

\begin{tabular}{lcccccc}
\hline & \multicolumn{5}{c}{ Calidad de atención percibida por usuarios } \\
\cline { 2 - 6 } Satisfacción Laboral & Global & Empatía & $\begin{array}{c}\text { Elementos } \\
\text { tangibles }\end{array}$ & Confiabilidad & $\begin{array}{c}\text { Capacidad } \\
\text { de respuesta }\end{array}$ & Seguridad \\
\hline Global & 0,062 & 0,063 & $0,122^{*}$ & 0,022 & 0,016 & $-0,002$ \\
Satisfacción & 0,015 & 0,011 & $0,111^{*}$ & $-0,024$ & $-0,028$ & $-0,038$ \\
Presión & 0,089 & 0,062 & $0,138^{* *}$ & 0,067 & 0,063 & 0,033 \\
Relación personal & 0,004 & 0,031 & 0,022 & $-0,004$ & 0,03 & $-0,019$ \\
Distensión & $0,157^{* *}$ & $0,124^{*}$ & $0,178^{* *}$ & $0,118^{*}$ & $0,106^{*}$ & $0,108^{*}$ \\
Adecuación & $-0,041$ & $-0,049$ & $-0,009$ & $-0,063$ & $-0,055$ & $-0,054$ \\
Control & $0,106^{*}$ & $0,155^{* *}$ & 0,075 & 0,048 & 0,075 & 0,085 \\
Variedad & $-0,073$ & $-0,067$ & $-0,115^{*}$ & $-0,106^{*}$ & $-0,117^{*}$ & $-0,112^{*}$ \\
\hline
\end{tabular}

Correlaciones significativas al 0,05 con $^{*}$ y al 0,01 con $^{* *}$.

Tabla 5. Niveles de SQT y Calidad de atención percibida por los usuarios, Servicios de Medicina y Cirugía, Hospital de RM de Chile.

\begin{tabular}{lcccccc}
\hline \multirow{2}{*}{$\begin{array}{l}\text { Factores de Síndrome } \\
\text { de Quemarse en el } \\
\text { Trabajo (SQT) }\end{array}$} & Global & Empatía & $\begin{array}{c}\text { Elementos } \\
\text { tangibles }\end{array}$ & Confiabilidad & $\begin{array}{c}\text { Capacidad } \\
\text { de respuesta }\end{array}$ & Seguridad \\
\hline Global & $-0,115^{*}$ & $-0,109^{*}$ & $-0,141^{* *}$ & $-0,078$ & $-0,043$ & $-0,063$ \\
Ilusión & 0,057 & 0,059 & $0,104^{*}$ & 0,019 & 0,018 & 0,006 \\
Desgaste & $-0,019$ & $-0,017$ & $-0,07$ & 0,004 & 0,004 & 0,037 \\
Indolencia & $-0,111^{*}$ & $-0,101^{*}$ & $-0,07$ & $-0,101^{*}$ & $-0,055$ & $-0,105^{*}$ \\
Culpa & $-0,154^{* *}$ & $-0,134^{* *}$ & $-0,159^{* *}$ & $-0,123^{*}$ & $-0,075$ & $-0,122^{*}$ \\
\hline
\end{tabular}

${ }^{\star}$ Se marcan las correlaciones significativas al 0,01 ** y las significativas al 0,05 con *.

\section{DISCUSIÓN Y CONCLUSIÓN}

A nivel nacional e internacional, el perfil demográfico de las enfermeras sigue siendo de predominio femenino en un $80 \%$ o más (25) y en este estudio ese porcentaje es mayor aún $(97,1 \%)$. Con relación al grupo etario, este estudio y lo que se reporta en Chile (52,9\%), es que los equipos de enfermería están ma- yoritariamente compuestos por menores de 34 años, cuestión que es diferente a la realidad mexicana o colombiana, en cuyos reportes son 38 y 43 años la edad promedio, respectivamente $(20,26)$. Respecto al estado civil, también existen diferencias. Mientras en otros países se reporta que la mayoría de las enfermeras son casadas $(61 \%)$ y con hijos $(20,26)$, en este estudio predominan enfermeras solteras y sin hijos. 
En el perfil laboral también existen diferencias, pues los estudios antes mencionados reportan que los años de trabajo en la institución en Colombia son 9,28 años y en México 14,6 años, y en este estudio, considerando que los años de ejercicio profesional son menos de 3 años en un $43 \%$ de la muestra, la permanencia en la institución que predomina con un $77 \%$ es entre uno y diez años (20, 26). Futuros estudios podrían indagar las razones de estas diferencias.

Respecto a las remuneraciones, los hallazgos del presente estudio son congruentes con otras investigaciones $(6,20,25-27)$ porque, en general, las enfermeras más jóvenes tienden a sentirse más satisfechas con sus remuneraciones, tanto en instituciones públicas como privadas.

Respecto a la satisfacción laboral en enfermeras(os), en este estudio el valor de la satisfacción media global (2,26 puntos) resultó ser menor que en otros estudios como en Colombia, que han alcanzado un valor medio de 3 puntos o cercanos a ese valor, 2,91 (28) y coinciden en que la dimensión mejor calificada es "la relación interpersonal" $(20,28,29)$. Se pudo observar también, que la dimensión "presión en el trabajo" era la que producía menor satisfacción laboral y esta diferencia se puede explicar en base a los lugares en que trabajaban esas enfermeras, que incluía unidades de cuidados intensivos, pediatría y urgencias, todos con una mayor complejidad e intensidad de la atención, una formación más especializada $y$, aunque dan la sensación de una mayor carga de trabajo para responder adecuadamente a estas demandas, finalmente proporcionan mayor satisfacción (28-30). Esto se refuerza con el comportamiento de la dimensión "adecuación con el trabajo" que aunque en este estudio fue un factor que produce satisfacción, en otras realidades como México (20) es una de las dimensiones con menor satisfacción. Esto se explicaría también por la diferencia en el perfil sociodemográfico de los diferentes tipos de servicios estudiados. En Chile se realizó en servicios de Medicina y Cirugía, donde las actividades cotidianas son rutinarias con complejidad moderada y por tanto requieren menos años de experiencia laboral, lo que conlleva a un menor nivel de satisfacción laboral global y mayor adecuación con el trabajo versus el estudio mexicano realizado en centros de especialidades, donde las tareas son complejas, difíciles y variadas, lo que produce mayor satisfacción laboral y menor adecuación para el trabajo (20). No en vano se han descrito como variables predictoras de la satisfacción laboral, el tiempo en la profesión y lugar en el que realiza su trabajo, siendo la de mayor peso el lugar en que trabajan (30).

Respecto al SQT, en un estudio realizado en Colombia que utilizó el mismo instrumento CESQT, presenta un resultado global bajo de SQT, coincidente con lo encontrado en este estudio (30). Ambos coinciden en que las dimensiones con menor SQT son "ilusión por el trabajo" "indolencia y culpa". Esto significa que las enfermeras(os) que se perciben autoeficaces, consideran su trabajo como atractivo y piensan que alcanzar las metas profesionales es una fuente de realización personal, no muestran insensibilidad y se conmueven ante los problemas de los pacientes. Con respecto al "desgaste psíquico", cabe destacar que en el estudio colombiano (30) se reportan niveles medios de desgaste psíquico, con valores más bajos en unidades de especialidades y cirugía, y más altos en el bloque de hospitalización y servicios generales. Esto último es coincidente con este estudio, pues la dimensión que presenta el mayor nivel de SQT es "desgaste psíquico" (7, 30 ), reportado por el $45,7 \%$ de las enfermeras(os). Esto se explica por la asociación que existe con dimensiones psicosociales como: carga laboral, contenido del cargo, exigencias laborales, desarrollo de carrera, interacción social y aspectos organizacionales (31-34).

$\mathrm{Al}$ analizar las expectativas y las percepciones de la calidad de atención percibida por los usuarios, se puede observar que las 
expectativas tienden a ser bastante altas y mayores que la percepción, lo que significa que los usuarios atendidos por los equipos de enfermeras estudiadas perciben una deuda en la calidad de atención recibida, ya que un servicio se considera de calidad cuando las percepciones igualan o superan las expectativas (35).

En un estudio de calidad de atención realizado en cuidadores principales de niños con limitación física en servicios de fisioterapia en un hospital de II nivel en Colombia, que utilizó el SEVQUAL, se evidenció que en todos las dimensiones existe una brecha entre las expectativas y las percepciones, lo que denota una tendencia a la insatisfacción, y esto es concordante con este estudio (35), porque las dimensiones que presentan mayor insatisfacción en el usuario, es decir, las con mayor brecha entre expectativas y percepciones, corresponden a "confiabilidad y empatía”. Esto significa que los usuarios perciben que la atención presenta déficit para realizar el servicio prometido de forma fiable y cuidadosa, y que falta un cuidado individualizado y disponibilidad para ponerse en el lugar del otro. Los con mayor satisfacción son "seguridad" y "capacidad de respuesta". Estas últimas dimensiones se refieren a la cortesía y habilidad del profesional para transmitir credibilidad, a la confianza y confidencialidad en la atención y a la disposición y buena voluntad de ayudar a los usuarios y proveerlos de un servicio rápido y oportuno.

La relación entre la satisfacción laboral Global de los equipos de enfermeras(os) y calidad de atención global percibida por los usuarios no presenta una correlación significativa. El análisis específico revela que las dimensiones de satisfacción laboral que más relaciones significativas presentan con calidad de atención son "variedad" y "distensión", es decir, a mayor variedad de la tarea, mayor (menor brecha) es la calidad de atención percibida por el usuario, y están relacionados con cuatro de cinco dimensiones evaluadas: "elementos tangibles" (apariencia personal, condiciones físicas, equipo e información), "confiabilidad" (proporcionar el servicio prometido con precisión y eficacia), "capacidad de respuesta” (ayudar y proporcionar el cuidado rápidamente de enfermería) y "seguridad” (inspirar confianza y credibilidad). No se encontró relación significativa con la dimensión "empatía” (atención individual y personalizada), sin embargo, en la segunda dimensión de calidad de atención percibida, a mayor capacidad de los equipos de enfermería para desconectarse del trabajo, poder hacer la tarea y tener la sensación de que el salario es adecuado, menor (mayor brecha) es la calidad de atención percibida por el usuario considerando todas las dimensiones de la calidad de atención.

Por otra parte, los análisis correlacionales muestran que a mayor SQT menor es la calidad de atención global y esto, junto con relacionarse con la "capacidad de los equipos de enfermería de ejecutar un cuidado de enfermería individual y personalizado", también se relaciona con la "apariencia personal", las "condiciones físicas", el "equipo e información”. Lo mismo ocurre entre la dimensión "indolencia" con calidad de atención global, "empatía", "confiabilidad" y seguridad".

Cabe destacar que la dimensión de SQT "culpa" aunque presenta una asociación estadísticamente significativa, es de magnitud muy baja en casi todas las dimensiones de calidad de atención percibida por los usuarios, porque la "capacidad de respuesta" fue un factor con el que no es significativa la correlación. Asimismo, la dimensión "desgaste psíquico” de SQT no presenta ninguna asociación estadísticamente significativa con calidad de atención, es decir, para esta muestra la dimensión "desgaste psíquico" es muy baja e independiente de la calidad de atención. Este resultado llama la atención considerando que en este estudio y en otros (30-32) la dimensión con más alto nivel de SQT es "desgaste psíquico". Esto puede tener su explicación en el tamaño de la muestra estudiada pues se sabe que ambientes favo- 
rables a la práctica profesional del equipo de enfermería pueden favorecer la existencia de menores niveles de agotamiento emocional, y por ende, mejor calidad del cuidado y una percepción positiva sobre actitudes de seguridad, en términos de equipo, clima de seguridad, satisfacción en el trabajo y comportamiento seguro (9).

Finalmente, cabe destacar que se identificaron dos limitaciones derivadas del instrumento que evalúa la calidad de atención percibida por los pacientes: a) el cuestionario era difícil de entender, está redactado igual para ambas secciones (expectativas y percepciones) y había palabras difíciles para los pacientes. La literacidad en Chile es baja $(11,5 \%)(36)$ y para hacer frente a esta limitación cada paciente recibía explicación al significado de los términos; $y \mathrm{~b}$ ) no se descarta que las respuestas pueden haber estado condicionadas por temor a repercusiones en su atención dado que se trataba de una evaluación del servicio, por eso las encuestas, una vez respondidas, eran depositadas por los mismos participantes en urnas selladas.

Los equipos de enfermeras(os) de los servicios de cirugía y medicina de un hospital de la RM de Chile presentan: 1) niveles de satisfacción laboral media, y las dimensiones que más contribuyen a elevar esa satisfacción son: "satisfacción con el trabajo", "distensión" y "relación interpersonal con los jefes"; 2) un SQT global bajo, siendo la dimensión "desgaste psíquico" el que más contribuye a ello.

Respecto a calidad de atención percibida por los usuarios de los servicios de Medicina y Cirugía de un hospital clínico de la RM de Chile, se puede decir que: 1 ) perciben una deuda en la calidad de atención recibida por los equipos de enfermeras(os); 2) está determinada por el SQT ya que a mayor SQT menor es la calidad de atención global y a esta correlación contribuyen la capacidad de los equipos de enfermería de ejecutar un cuidado de enfermería individual y personalizado y también la apariencia personal, las condiciones físicas y el equipo e información; 3)
No se relaciona con la satisfacción laboral global de los equipos de enfermeras(os).

Los análisis correlacionales globales y por dimensiones realizados entregan una información valiosa para tomar decisiones específicas que permitan mejorar en forma más efectiva la satisfacción laboral, el SQT y la calidad de atención percibida por los usuarios.

Se sugiere continuar con estudios en esta línea dirigidos a este grupo de profesionales de la salud, con una muestra más amplia que permita establecer correlaciones significativas más altas que profundicen en las dimensiones y permitan validar estos resultados en el país.

\section{REFERENCIAS}

1. Díaz F, Gómez I. La investigación sobre el Síndrome de Burnout en Latinoamérica entre el 2000-2010. Psicología desde el Caribe. 2016; 33(1): 113-131.

2. Figueiredo- Ferraz H, Grau-Alberola E, Gil-Monte P, Garcia- Juesas J. Síndrome de quemarse por el trabajo y satisfacción laboral en profesionales de enfermería. Psicotema. 2012; 24(2): 271-276.

3. Hill A, Curran T. Multidimensional Perfectionism and Burnout: A Meta-Analysis. Pers Soc Psychol Rev. 2016; 20(3): 269-288.

4. Portero de la Cruz S., Vaquero M. Professional burnout, stress and job satisfaction of nursing staff at a university hospital Rev Lat Am Enfermagem. 2015; 23(3): 543-552.

5. Girbau M, Galimany J, Garrido E. Desgaste profesional, estrés y abandono de la profesión en enfermería. Nursing. 2012; 30(1): 58-61.

6. Ruzafa-Martínez M, Madrigal-Torres M, Velandrino-Nicolás A, López-Iborra L. Satisfacción laboral de los profesionales de enfermería españoles que trabajan 
en hospitales ingleses. Gac Sanit. 2008; 22(5): 434-42.

7. Rodwell J, Munro L. Well-being, satisfaction and commitment: the substitutable nature of resources for maternity hospital nurses. J Adv Nurs. 2013; 69(10): 22182228.

8. Aiken LH, Sloane DM, Bruyneel L, Van den Heede K, Griffiths P, Busse R et al. Nurse staffing and education and hospital mortality in nine European countries: a retrospective observational study. Lancet. 2014 ; 383(9931): 1824-1830.

9. Guirardello EB. Impact of critical care environment on burnout, perceived quality of care and safety attitude of the nursing team. Rev Lat Am Enfermagem [Internet]. 2017 [citado 17 nov 2017]; 25: e2884. Disponible en: http://dx.doi. org/10.1590/1518-8345.1472.2884

10. Hayes LJ, O’Brien-Pallasa L, Duffield C, Shamian J, Buchan J, Hughes F, et al. Nurse turnover: A literature review. Int J Nurs Stud. 2006; 43(2): 237-263.

11. Zeytinoglu IU, Denton M, Davies S, Baumann A, Blythe J, Boos L. Retaining nurses in their employing hospitals and in the profession: Effects of job preference, unpaid overtime, importance of earnings and stress. Health Policy. 2006; 79(1): 5772.

12. Garrosa E, Moreno-Jiménez B, Liang Y, González JL. The relationship between socio-demographic variables, job stressors, burnout, and hardy personality in nurses: An exploratory study. Int J Nurs Stud. 2008; 45(3): 418-427.

13. Barrios S, Arechabala MC, Valenzuela V. Relación entre carga laboral y burnout en enfermeras de unidades de diálisis. Enferm Nefrol. 2012; 15(1): 46-55.

14. De Tienne K, Agle B, Phillips J, Ingerson M. The Impact of Moral Stress Compared to Other Stressors on Employee Fatigue, Job Satisfaction, and Turnover: An Empirical Investigation. J Bus Ethics 2012; 110(3): 377-391.
15. Austria F, Cruz B, Herrera L, Salas J. Relaciones estructurales entre estrategias de afrontamiento y síndrome de Burnout en personal de salud: un estudio de validez externa y de constructo. Univ Psychol. 2012; 11(1): 197-206.

16. Jofre V, Valenzuela S. Burnout en personal de enfermería de la unidad de cuidados intensivos pediátricos. Aquichan. 2005; 5(1): 56-63.

17. Massip C, Ortiz R, Llantá M, Peña M, Infante I. La evaluación de la satisfacción en salud: un reto a la calidad. Rev Cub Salud Pública [Internet]. 2008 [citado 9 abr 2013]; 34(4) Disponible en: http://scielo. sld.cu/scielo.php?script=sci_arttext\&pi$\mathrm{d}=$ S086434662008000400013\&lng=es

18. Ministerio de Salud. Subsecretaría de Redes Asistenciales. Medición Nacional de Satisfacción usuaria en la Red pública de Salud de Chile. Aplicación 2009 [Internet]. Santiago, Chile: Serie Cuadernos de Redes No 32, Subsecretaría de Redes Asistenciales; 2009 Dic [citado 20 ago 2013]. 102 p. Disponible en: http://www. bibliotecaminsal.cl/wp/wp-content/ uploads/2016/03/32.pdf

19. Aranaz J, Mira J. Cuestionario Font Roja. Un instrumento de medida de la satisfacción en el medio hospitalario. Todo hospital. 1988; 52: 63-68.

20. Hernández M, Hernández A, Nava M, Pérez M, Hernández M, Matus R, et al. Satisfacción laboral del profesional de enfermería en cuatro instituciones de salud. Enferm. univ. 2012; 9(1): 7-15.

21. Olivares V, Gil-Monte P. Prevalencia del síndrome de quemarse por el trabajo (burnout) en trabajadores de servicios en Chile. Informació Psicològica, 2008; 9192: 43-52.

22. Parasuraman A, Berry LL, Zeithaml VA. Refinement and Reassessment of the SERVQUAL Scale. Journal of Retailing. 1991; 67(4): 420-50.

23. Jelvés A, Riquelme Y, Gómez N. Evaluación de la calidad de servicio en centro de 
salud familiar en Chile. Horizontes Empresariales. 2010; 9(2): 51-72.

24. Pineda U, Estrada M, Parra C. Aplicación del modelo SERVQUAL y herramientas de ingeniería de la calidad para la planificación del servicio en la Biblioteca Central de la Universidad de Antioquia. Rev. Interam. 2011; 34(3): 243-255.

25. Ministerio de Salud, Chile. Informe sobre brechas de personal de salud por servicio de salud [Internet]. Santiago, Chile: Subsecretaría de Redes Asistenciales, División de Gestión y Desarrollo de las Personas, Departamento de Planificación y Control de Gestión de RHS; 2017 Abr [citado 24 enero 2017]: 88 p. Disponible en: http://www.minsal. cl/wp-content/uploads/2015/08/Informe-Brechas-RHS-en-Sector-P\%C3\%BAblico_Abril2017.pdf

26. Molina Linde JM, Avalos Martínez F, Valderrama Orbegozo LJ, Uribe Rodríguez AF. Factores relacionados con la satisfacción laboral de la enfermería de un hospital médico-quirúrgico. Invest Educ Enferm. 2009; 27(2): 218-225.

27. Rodríguez R, Fonseca D, Castro E, Betancourt I. Satisfacción laboral en enfermeros del Instituto de Nefrología. INFODIR. 2012; 8(14): 25-29.

28. Cifuentes J, Manrique F. Satisfacción laboral en enfermería en una institución de salud de cuarto nivel de atención, Bogotá, Colombia. av. enferm. 2014; XXXII(2): 217-227.

29. Rodríguez J. Satisfacción Laboral y síndrome de Burn Out en el personal de enfermería Hospital Nacional Psiquiátrico. Enfermería en Costa Rica. 2012; 32(2): 4-10.

30. González L, Guevara E., Morales. G., Se- gura P., Luengo C. Relación de la satisfacción laboral con estilos de liderazgo en enfermeros de hospitales públicos, Santiago, Chile. Cienc. enferm. 2013; XIX(1): 11-21.

31. Toro D. Nivel de burnout en profesionales de enfermería en una institución prestadora de servicios de tercer nivel de la ciudad de Manizales [Tesis de Magíster]. [Manizales (CO)]: Universidad Nacional de Colombia; 2014.90 p.

32. Molina J, Avalos F, Giménez I. Burnout en enfermería de atención hospitalaria Enfermería Clínica. 2005; 15(5): 275-282.

33. Aldrete MG, Navarro C, González R, Contreras M, Pérez J. Factores psicosociales y síndrome de burnout en personal de enfermería de una unidad de tercer nivel de atención a la salud. Cienc Trab. 2015; 17(52): 32-36.

34. Borré Y, Vega Y. Calidad percibida de la atención de enfermería por pacientes hospitalizados. Cienc. enferm. 2014; XX(3): 81-94.

35. Salazar-Villamarín C, Gómez-Ramírez, E., Zuluaga, M. A. Calidad percibida de los cuidadores principales de niños con limitación física sobre los servicios de fisioterapia en un hospital de II nivel en Colombia. Memorias. 2015; 13(23): 7-19.

36. Ministerio de Salud Chile. Encuesta nacional de salud 2016-2017. Primeros resultados [Internet]. Santiago, Chile: Departamento de Epidemiología. División de Planificación Sanitaria. Subsecretaría de Salud Pública; 2017 nov [citado 21 nov 2017]. Disponible en: http://epi. minsal.cl/wp-content/uploads/2017/11/ ENS_2016-17_PRIMEROS-RESULTADOS.pdf 